\title{
Coping: Self-Deception in Action
}

\author{
James F Welles* \\ PO Box 17, East Marion, New York, USA \\ *Corresponding author: James F Welles, P O Box 17, East Marion, New York, USA
}

Received: 泚 January 07, 2020

Published: 留 January 21, 2020

\section{Mini Review}

When people interact with their environment, their behavior is directed by a schematic cognitive program. A particular act can be construed as "Intelligent" or "Stupid" depending upon the perceived degree of success achieved, but while these labels indicate opposite evaluations, they do not indicate different cerebral programs. Nor should stupidity be viewed as a disruption of an "Intelligence mechanism". There is a coping (or responding) mechanism in action, and it can be construed as stupid and/or intelligent depending upon the circumstances and the judges. This coping mechanism is multidimensional, but we shall focus on three arbitrary/subjective facets important to understanding stupidityinformation processing, (mal)adaptation and relevance [1].

When considering the ways by which the human mind processes information, it is imperative to remember that the normal cognitive state is that of self-deception. Our self-deceptive nature tends to make us stupid and, more to the point of our analysis here, certainly complicates the relationship of knowledge to stupidity. If people simply do not have relevant information available to them in a perceivable form, they are agnostic. However, if they ignore available information to the impairment of schematic accuracy, they are being self-deceptive and probably stupid. Likewise, if they misinterpret information, they are being "Data dumb", although there may be some social advantage to certain cognitive indiscretions. The person who ignores warnings of an impending disaster exemplifies the condition of being data dumb. Military history, particularly, provides a litany of warnings unheeded or misconstrued [2].

The relationship of knowledge to stupidity is very circumstantial. Usually, the more one knows about a situation, the more successful his behavior is likely to be, but there is certainly no advantage in being overloaded with useless information. Worse yet, a person may worry himself sick if he is un-fortunate enough to know about a threatening situation over which he has no influence what-so ever. Thus, having knowledge can be maladaptive, particularly if one has no coping response available.

If the relationship between stupidity and knowledge is mostly circumstantial, that be-tween stupidity and ignorance is usually reciprocal. Ignorance often exists because a schema blocks relevant learning. On the other hand, stupidity may keep people ignorant by inhibiting behavior which would allow corrective, adaptive learning. Instead, a positive feedback system may then make behavior increasingly maladaptive to the immediate environment [3].

Data processing systems are most mal-adaptive when they make dysfunctional associations among bits of information. Stupidity is thus made more likely when there is not enough information (a party is to some degree uninformed), when there is too much (overloaded) but most commonly when it is wrong (misinformed). Stupidity also results when in-formation that is present and correct is misemphasized or misinterpreted. Of course, more profound kinds of stupidity are produced from a complexing of different possible source errors e.g., a misinformed person misinterpreting inaccurate data. Finally, the process be-comes blatantly unethical when a person deliberately cherry picks facts from an ambiguous field of data to rationalize a predetermined course of action [4-6].

Just as many factors related to information processing may render a schema maladaptive, so is the determination of "Maladaptation" another very arbitrary/subjective facet of the general coping mechanism of the mind. For example, although a person may know his drug addiction is maladaptive over the long haul, getting the next fix is most compelling and in his immediate, short-term best interest. While it may be to a particular company's advantage to control more than a fair share of resources, this may be maladaptive for its supporting culture. Since determining mal adaptivity depends so much on the arbitrary selection of the referent time scale and the standards and perspectives for judgment as well as the subjective evaluations of the judges, it, like "Knowing", turns out to be a rather imprecise guide for determining whether or not an act is to be deemed stupid [7].

When attempting to determine whether an act is adaptive or maladaptive, subjective judgments may be predetermined by the arbitrary selection of the referent itself. Is behavior maladaptive for an individual? His reference group? The environment? Behavior can be maladaptive relative to any or all of these referents. A 
human system virtually by definition is internally inconsistent and maladapted to itself. It can pointedly disrupt communication and adjustment to other human systems, and it can prevent accurate feedback from the environment, to the long-term detriment of the capacity of nature to sustain the human experiment. In this context and as a refutation of the theory of cognitive dissonance (cogdis), Adolf Hitler was a case study in stupidity in that the more his belief in Providence (i.e., God) was contradicted by facts from his environment, the stronger he embraced it -clearly a road map for a disastrous one-way, dead-end street.

Cognitive dissonance is defined as an uncomfortable condition resulting from holding contradictory beliefs. Obviously, stupidity helps in this process, in that the dumber a person is, the easier it is for her to hold contradictory beliefs. For us, we expand cogdis to cover the condition on a deeply held belief at odds with relevant behavior as in Crusaders who killed for Christ or a corrupt cop. In addition, we extend the principle beyond the individual to society in general, in that we find a culture may profess a belief which is contradicted by the conduct of its members, as when the institution of science is set up to protect errant members rather than correct their errors [8].

While it is reasonable to presume that a such dissonance creates a psychic tension which humans ache to resolve, this commonly is not the case: people simply live with their contradictions. As Walt Whitman observed, "Do I contradict myself? Very well then. I contradict myself." This is not much of a stretch, since one can posit that a behavioral system is an expression of an underlying belief system-so you have a behavioral belief system at odds with a theoretical/ethical belief system. The expected dissonance is common mitigated by-word games, which reduce cognitive tension by redefining everything to suit the super-ego individual and society. The oddity is that people then get upset when confronted by some aggravating person who, like the honest cop, presumes to act according to the explicit verbal creed and challenges the society to adapt to her, live up to itself or admit its basic ethic is a not particularly amusing self-inflicted joke [9].

Generally, in any situation, there are three concentric fields for behavioral adaptation. The first is an individual system a person, business group, team, etc. The next is the social context of the supporting culture other individuals and groups. Finally, there is the ultimate arbiter of fitness the physical environment. An intelligent policy is one which is advantageous to the performer, beneficial to humanity in general and at least not detrimental to the environment. The development of the telephone might serve as an example of an invention which was a success all three ways. Mr. Bell and his family prospered; society was provided with speedier communication; and, except for some unsightly wires, no major negative impact on the environment was suffered. Usually, of course, a policy engenders new problems as it solves the old by emphasizing success in the first, limited category at the expense of the others [10].

Thus, can a policy be both adaptive and maladaptive. In a short time span a pattern of behavior can be construed as adaptive by those who profit from it while it is condemned by those who must endure it. Over a longer time span, individuals may alter their judgments about a policy as they become aware of unexpected and clearly negative results. As a bottom line, "Self-interest" is really the final criterion of judgment, and stupidity is behavior counterproductive to the welfare of the performer. As the Shah of Iran, Ferdinand Marcos of the Philippines and Nicolae Ceausescu of Romania found, the immediate pursuit of one's own best interest may be, in the long run, neurotic-paradoxically maladaptive in the extreme [11].

The American industrial complex is a prime example of a dynamic association of similar organizations concentrating on their own short-term enhancement while contributing to the demise of the common life support system for general society. The government's response to the pollution and exploitation of our natural resources was the Environmental Protection Agency. On non-recycled paper, it was an ideal solution to a real problem. In reality, it was taken over by the industries it was designed to control. Its record in promoting pollution and the desecration of nature is unsurpassed in the annals of government. It is most easily dismissed as a misnomer: It should be called the Industrial Protection Agency or the Environmental Pollution Agency.

If it is difficult to generalize about and define maladaptation, it may be quite easy to recognize. Commonly, a behavioral trend goes to a self-defeating excess. Technological over-development, political repression and human exploitation are all examples of maladaptation induced by the inherent tendency of cultures to function as positive feedback systems. Such excesses usually indicate a power structure caught up in the neurotic paradox: excesses are promoted as entrenched values both reinforce established patterns of behavior and render criticism less likely and less effective. In most cases, a dominant subgroup controls its supporting culture and may be living beyond the carrying capacity of the general society.

Maladaptation usually indicates that the coping mechanism really is not "Coping" but is rather simply responding in counterproductive ways. In a more common but less spectacular fashion, nonadaptive behavior indicates that the coping mechanism is responding in wasteful, irrelevant (i.e., stupid) ways. As indicated above, the determination of relevance/irrelevance requires both arbitrary decisions and subjective judgments. The arbitrary criteria by which relevance may be subjectively judged are: context, personnel (the people who act/judge) and purpose.

The context of an object or behavior does much to determine -indeed, it practically defines-just what its relevance is. With regard to an object, as primatologist Winston S. Churchill observed: "A baboon in a forest is a matter of legitimate speculation; a baboon in a zoo is an object of public curiosity; but a baboon in your wife's bed is a cause of the gravest concern." As with baboons, so with behavior. Thus, the purpose of a doctor asking, "How are you?" may vary with the setting. In his office, it is likely a professional inquiry: on the street, it is probably a cultural throw-away.

Of course, context is not merely a matter of physical location. Behavior is invariably interpreted in a conceptual context, but it is 
the observer who arbitrarily selects the context in which relevance is judged. Thus, a patriotic hawk construes a Congressional vote for a large defense budget as laudable, whereas a frugal-minded economist would regard the same act as fiscal madness. The one views the purchase of vast amounts of military hardware and the maintenance of a sizable military force as necessary for national security; the other considers the money spent as an intolerable drain on our financial resources. Likewise, in legal affairs, the context may determine the legality of an act: exercises in freedom of speech in peacetime may be regarded as seditiously criminal in wartime.

In a similar way, arbitrary personal considerations play a major role in evaluating the relevance of behavior. The critical factor is the relationship between the actor and the observer. If a friend and an enemy do the same thing, two different interpretations are likely. In terms of the example just cited, a political ally voting for a large defense budget is patriotic, whereas an opponent doing so is a reckless spendthrift. Likewise, when a member of one's political party violates security by leak-ing evidence of official corruption, it is an act of courage; when a member of the opposition does so, it is a breach of security. (When a no-account enlisted man in the armed forces does it, it is a crime).

In addition to the three elements of subjectivity just considered, there is "Timing" -in the sense that for information processing to be helpful, it must be accomplished within a given time frame: the military call it "Actionable intelligence". This consideration is complicated by the fact that there is a built-in lag in all data processing, so some delay has to be accepted as a given. This might be measured in nanoseconds in the cases of computers, milliseconds in very sharp human minds or years in behaviorists, but there is some delay between perception and comprehension. If the delay is so short that adaptation is impossible, then the issue of stupidity does not arise. However, it is at best sad when one realizes too late what the available data "Really meant", and what one should have done.

To carry this a step further, there can be a stupidity lag between the moment one realizes what should be done and the time of action. If action is both timely and proper, it probably is successful. However, if it is delayed too long, even the correct response may be stupid. For an individual, the time scale might be measured in seconds while for nations, it might be years: as H. G. Wells observed, the English rely on a time lag of about 75 years between the perception that something should be done about a problem and a serious attempt to do it.

To confuse matters more, in times of decision while under psychological duress, meanings of words may change. This malaise afflicted Athens during the Peloponnesian War (431-404 B.C.) when reckless audacity in an ally became courage; prudent hesitation was cowardice; moderation was unmanly whereas frantic violence was the Greek equivalent of macho; and the liberal ability to see all sides of an issue was equated with the Hamletesque inability to act at all.

Not only does thinking become muddled in such circumstances, but there is also a cultural loss when attention afforded an innovation and its considered worth (relevance) are both only secondarily determined by its inherent worth. The status of the innovator may either add to or detract from the value an offering is accorded. This social dimension is a major determinant in groupthink: e.g., when a respected leader sponsors a proposition, it is likely to receive a favorable reaction from his followers.

The final criterion by which relevance is judged is that of purpose. Survival is a basic purpose of life, but when it becomes an end in itself, development ceases and is replaced by stagnation. When the purpose is simply to survive, human behavior is shaped by an opportunistic schema which is consistent only in the ease with which it yields to immediate circumstances. In such cases, life is a struggle for short-term existence, with no consideration for long-term ramifications of behavior. Such a schema might be labeled "Meism/ Nowism" as any other morality is an unaffordable luxury.

If behavior is not dictated by necessity, purpose can be created by a commitment to group norms. Accepting group standards can be stupid in that it defines adjustment in terms of a single, totally arbitrary value system. In general, most schemas are directed toward maintaining a status quo. Unfortunately, they may be so committed to themselves that they self-destruct. The process begins when an initially successful pattern of behavior becomes routine; when it serves to block innovation, it promotes failure. Reformers who then call for improvements in the schema are regarded as a source of distress. They are usually considered maladjusted and are not, in fact, adjusted to the cultural values society has enshrined as sacred. This particular kind of vexation is a growing problem today, as Western Civilization moves from making people equal in rights to similar in thought and behavior.

Finally, judgment may be complicated by the application of different schemas by different people to the same data. This occurred in November of 1983 when events sur-rounding a NATO exercise led to a threat of the Cold War turning hot. One of the problems was that the West viewed events in the context of Munich, 1938, when appeasement led eventually to war while the Soviets viewed the same events in the context of the German invasion of Russia in 1941, when they were caught off guard. This was a very real case of the application of different schemas leading to a very tense situation but fortunately nothing more, although matters were further complicated by President Reagan's disturbing personal belief that the confluence of events presaged Biblical Armageddon.

\section{References}

1. Lockard J, Paulhus P (Eds) (1988) Self-deception: An Adaptive Mechanism? Prentice-Hall, Englewood Cliffs, New Jersey, USA.

2. Schramm P (1999) Hitler: The Man \& the Military Leader. Academy Chicago; Chicago, USA.

3. Welles J Spring (2009) Correcting Errors in Science: An Analytic Review. Journal of Information Ethics. 18(1):16-20. Opposed to cogdis is F Scott Fitzgerald's (1936) insight that the mark of first-rate mind is the ability to hold contradictory ideas at the same time and still function. For my money, Festinger is being superegoish: His view is the way a reasonable mind should function. He and his have gathered a lot of supporting data, but to maintain their theory, they have to ignore a lot of street behavior which contradicts their view. The test set up by scientists show how 
the mind can function-not necessarily how it does func-tion. More perplexing and confusing is the fact that even a first-rate mind can function well with just a half-baked idea if it is gratifying. (Dolnick. The Seeds of Life. Basic Books, New York. 2017. 251.)

4. Whitman W (1855) Song of Myself.

5. Tuchman B (1984) The March of Folly. Knopf, New York, USA

6. Halle K (1967) The Irrepressible Churchill: A Treasury of Winston Churchill's Wit. World Publishing, New York, USA pp. 153.
7. John R Vile (1917) Masses Publishing Co. v. Patten. pp. 535-541.

8. Gup T (2007) Nation of Secrets. Doubleday, New York, USA, p. 63.

9. Wells H (1931) The Work, Wealth and Happiness of Mankind. Chapter 11.

10. Thucydides. Ca. 410 B.C. History of the Peloponnesian Wars.

11. Janis I (1982) Groupthink. Houghton Mifflin, Boston, USA, pp. 142.

\section{(C) (P) \\ This work is licensed under Creative Commons Attribution 4.0 License}

To Submit Your Article Click Here:

Submit Article

DOI: $10.32474 / J A A S .2020 .01 .000113$

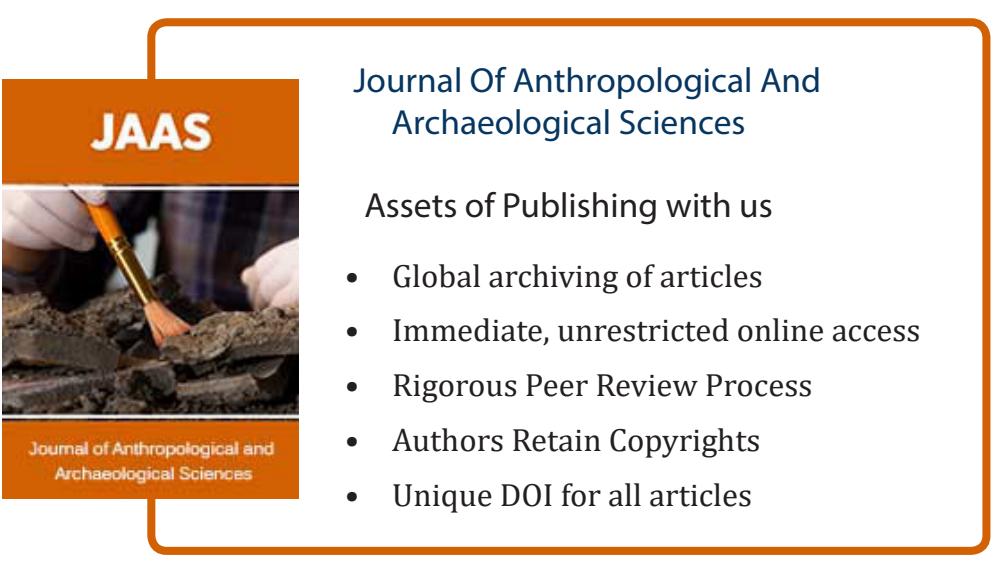

